\title{
The Influence of Monomer Composition and Surface-CrossLinking Condition on Biodegradation and Gel Strength of Super Absorbent Polymer
}

\author{
Jung Soo Kim ${ }^{1,2}$, Dong Hyun Kim ${ }^{2}$ and Youn Suk Lee ${ }^{1, *(\mathbb{D})}$ \\ 1 Department of Packaging, Yonsei University, Wonju 26493, Korea; kimjungsoo11@kitech.re.kr \\ 2 Human Convergence Technology R\&D Departments, Korea Institute of Industrial Technology (KITECH), \\ Ansan 15588, Korea; dhkim@kitech.re.kr \\ * Correspondence: leeyouns@yonsei.ac.kr; Tel.: +82-33-760-2395
}

check for updates

Citation: Kim, J.S.; Kim, D.H.; Lee, Y.S. The Influence of Monomer Composition and

Surface-CrossLinking Condition on Biodegradation and Gel Strength of Super Absorbent Polymer. Polymers 2021, 13, 663. https://doi.org/ $10.3390 /$ polym 13040663

Academic Editor:

George Z. Papageorgiou

Received: 29 December 2020

Accepted: 16 February 2021

Published: 23 February 2021

Publisher's Note: MDPI stays neutral with regard to jurisdictional claims in published maps and institutional affiliations.

Copyright: (c) 2021 by the authors. Licensee MDPI, Basel, Switzerland. This article is an open access article distributed under the terms and conditions of the Creative Commons Attribution (CC BY) license (https:/ / creativecommons.org/licenses/by/ $4.0 /)$.
Abstract: In this study, a superabsorbent polymer (SAP) comprising poly (IA-co-cellulose-co-VSAco-AA; ICVA) core-SAP (CSAP) was synthesized through radical polymerization using itaconic acid (IA), acrylic acid (AA), cellulose, and vinyl sulfonic acid (VSA) as monomers. The absorption performances and relative biodegradability of various compositions prepared by adjusting the amounts of cellulose and VSA with constant IA and AA content were compared. Increasing the cellulose content in CSAP contributed to improved biodegradation of the surface-crosslinked SAP (SSAP) and gel strength, although the free absorbency (FA) and centrifuge retention capacity (CRC) decreased. Increasing the VSA content resulted in strong anionicity, which enables the absorption of large amounts of water. Surface-crosslinking technology was applied to the CSAP synthesized with the optimal composition ratio to increase its absorption performance and gel strength. Improved performance of the synthesized SSAP (a CRC of $30.4 \mathrm{~g} / \mathrm{g}$, absorbency under load (AUL) of $23.3 \mathrm{~g} / \mathrm{g}$, and permeability of $55 \mathrm{~s}$ ) was achieved by selecting the optimal surface-crosslinking treatment time and the amount of distilled water in the surface-crosslinking solution: as the latter was increased in the surface-crosslinking solution, the AUL and permeability of the SSAP were improved, and its biodegradability was found to be $54 \%$ compared to the $100 \%$ biodegradable cellulose hydrogel in the control group.

Keywords: superabsorbent polymer; biodegradable; surface-crosslinking; itaconic acid; cellulose

\section{Introduction}

A superabsorbent polymer (SAP) can absorb water at tens to hundreds of times its own weight and does not easily release water under a certain pressure. Due to these characteristics, they are most commonly used in sanitary products such as diapers for infants and adults, absorbent pads and packaging materials for food distribution, water-retaining soil and seedling sheets in agriculture and horticulture, and drug delivery materials for pharmaceuticals [1-3].

Recently, many studies have been conducted to improve both permeability and absorbency under load (AUL) by increasing the gel strength while maintaining other water absorption performance for application to infant disposable diapers. Normally, commercial SAPs used for disposable diapers have a centrifuge retention capacity (CRC) of about $30 \mathrm{~g} / \mathrm{g}$ and AUL of about $20 \mathrm{~g} / \mathrm{g}$ when measured using the EDANA (European Disposables and Nonwovens Association) method [4]. The gel strength can be enhanced by increasing the density of the internal and surface-crosslinking. However, increasing the gel strength narrows the network width and reduces absorption capacity at the same time. In this trade-off relationship, it is important to select the appropriate monomers and find the optimal polymerization conditions [5-8]. 
SAPs, which show excellent absorption characteristics, are generally synthesized using petroleum-based monomers such as acrylic acid (AA) or acrylamide. However, they have disadvantages such as being toxic to the skin and are environmentally unfriendly $[9,10]$. Accordingly, many researchers have studied the synthesis of SAPs using bio-based monomers to reduce the content of petroleum-based monomers such as AA and acrylamide [11-16]. Typically, potato starch, cassava starch, corn starch, and cellulose have been widely used in biomass-based SAP synthesis because these are inexpensive and widely available. Replacing SAP production using petroleum-based monomers with starch-based monomers can reduce the final cost and improve the biodegradability of the materials. Biomass-based SAP has excellent biodegradation properties, but has limitations in absorption performance such as gel strength. Accordingly, biomass-based SAP has poor performance as a diaper material, and has mainly been used as an agricultural material.

Itaconic acid (IA), which is often used as a biodegradable monomer, is an unsaturated dicarboxylic acid that is stable at room temperature and easily degrades into soil because it is prepared by cultivating bacteria in sugarcane badges. Furthermore, IA can be radically polymerized with vinyl monomers. Therefore, it can replace petroleum-based AA. Moreover, IA has two carboxylic acid groups and is water-soluble, and it has been selected by the US Department of Energy as the "most valuable substance in biomass" [17]. Since IA can be used as a component in SAPs, it can be used to create new biomass-based polymers that can mitigate some environmental problems [18].

Cellulose is a natural polysaccharide formed by plant photosynthesis and provides biological properties such as biocompatibility and nontoxicity [19]. Cellulose can improve hydrophilicity due to its hydroxylic groups, which can also contribute to improved gel strength $[20,21]$. However, most biomass-based SAPs have low gel strength, so their uses in biopharmaceuticals and agriculture are limited [22-24].

Vinyl sulfonic acid (VSA) is known to be hydrophilic and has high absorption performance. It has been reported that VSA is beneficial for gel expansion by inducing a reaction force between the polymer chains through its negatively charged sulfonate groups [25]. Accordingly, VSA has been widely used as a comonomer in copolymerization studies to improve SAP performance [26-28].

The most frequently applied technology in SAP studies to increase gel strength is surface-crosslinking. This produces additional networks bonded to a solid surface by reacting with functional groups that have survived on the core-SAP (CSAP) surface. The aim of surface-crosslinking technology is to enhance the strength of a gel when it is absorbing large amounts of liquid, and various parameters can be adjusted to improve its performance [29-31]. Gasri et al. reduced the surface-crosslinking treatment time of SAPs using a microwave method, studied the effects of $N, N$-dimethylaniline as a catalyst, and compared their performances according to the type and amount of surface-crosslinking agent [32]. Ha et al. produced AA-based SAPs containing different amounts of ethylene glycol diglycidyl ether and measured the thicknesses of their surface-crosslinked layers using a fluorescent dye [33]. Lee et al. compared the performances of AA-based SAPs according to surface-crosslinking time and the amount of polyethylene imine used as a surface-crosslinking agent: the thickness of the surface-crosslinked layer increased, and the absorbency decreased with increased surface-crosslinking time [34].

In this study, we confirmed the change in absorption properties according to the monomer composition and surface-crosslinking conditions in order to prepare SAP that has biodegradability and the performance required for diapers. Biomass-based IA and cellulose were used to enable biodegradation, and VSA having strong polarity and AA having excellent hydrophilicity were used as monomers to complement the absorption performance $[35,36]$. There is no study on an IA and cellulose combined SAP, which has better absorption performance, compared with existing eco-friendly SAP. The surfacecrosslinking density of the synthesized CSAP was further strengthened by esterification between hydroxide carboxyl groups on its surface and hydroxide groups on the surfaces of the surface-crosslinking agents. In addition, optimal conditions were determined by 
adjusting the surface-crosslinking treatment time and the amount of distilled water in the surface-crosslinking solution, after which the absorbency, gel strength, permeability, and biodegradability were evaluated.

\section{Materials and Methods}

\subsection{Materials}

Itaconic acid (IA, Junsei, special grade, Tokyo, Japan) and vinyl sulfonic acid (VSA, Sigma Aldrich, St. Louis, MI, USA), acrylic acid (AA) were used as a monomer without purification. Cellulose (CMC, Daejung, $\mathrm{M}_{\mathrm{w}}$ : 21,000-500,000, density: 1.59, Siheung, Korea) was purchased and used without purification. Poly(ethyleneglycol) diacrylate (PEGDA, Sigma Aldrich), 1,6-hexanediol diacrylate (HDODA, Sigma Aldrich) were used as the inner-crosslinker, sodium persulfate (NaPS, Sigma Aldrich) was used as the initiator, and 1,4-butanediol (BD, Sigma Aldrich, 99\%) was used as the surface-crosslinker without purification.

\subsection{Preparation of the CSAP}

IA, cellulose, VSA, and AA were added to the total content of $100 \mathrm{~g}$ in a four-neck reactor and $80 \mathrm{~g}$ of distilled water was added to maintain a nitrogen atmosphere (Table 1). A $50 \% \mathrm{NaOH}$ aqueous solution was slowly added to the reactor until it comprised $70 \%$ of the total molar ratio of total monomers. After the temperature was stabilized to less than $50{ }^{\circ} \mathrm{C}, 0.2 \mathrm{~g}$ of HDODA and PEGDA were added as inner-crosslinker and mixed uniformly. Thereafter, $0.5 \mathrm{~g}$ of NaPS was added to the reactor and stirred for $2 \mathrm{~h}$. Then, the reaction was transferred to a plastic tray and dried in a vacuum oven at $60^{\circ} \mathrm{C}$ for $24 \mathrm{~h}$. The dried product was frozen in a $-20^{\circ} \mathrm{C}$ freezer and then pulverized with a grinder. The crushed product was spread evenly on a wide tray and dried at $60^{\circ} \mathrm{C}$ for $12 \mathrm{~h}$. The dried product was classified into a particle size of 300 to $600 \mu \mathrm{m}$. We showed synthetic composition of CSAP and abbreviation of SAP made of poly(IA-co-cellulose-co-VSA-co-AA; ICVA).

Table 1. Synthetic composition of CSAP (core-superabsorbent polymer (SAP)).

\begin{tabular}{|c|c|c|c|c|c|c|c|}
\hline \multirow{2}{*}{ NAME * } & \multicolumn{4}{|c|}{ Monomer (g) } & \multicolumn{2}{|c|}{ Crosslinker (g) } & \multirow{2}{*}{$\begin{array}{c}\text { Initiator (g) } \\
\text { APS }\end{array}$} \\
\hline & IA & Cellulose & VSA & $\mathbf{A A}$ & HDODA & PEGDA & \\
\hline IVA & & 0 & 10.0 & & & & \\
\hline $\mathrm{IC}_{2.5} \mathrm{VA}$ & & 2.5 & 7.5 & & & & \\
\hline $\mathrm{IC}_{5.0} \mathrm{VA}$ & 50 & 5.0 & 5.0 & 40 & 0.25 & 0.25 & 0.5 \\
\hline $\mathrm{IC}_{7.5} \mathrm{VA}$ & & 7.5 & 2.5 & & & & \\
\hline ICA & & 10.0 & 0 & & & & \\
\hline
\end{tabular}

\subsection{Preparation of the Surface-Crosslinked SAP (SSAP)}

Table 2 shows the composition of the surface-crosslinking solution and surfacecrosslinking time for the preparation of SSAP. Solvent (methanol + distilled water) and $\mathrm{BD}$ were mixed in a ratio of 10:1 to prepare a surface-crosslinking solution in a $20 \mathrm{~mL}$ vial. CSAP ( $3 \mathrm{~g}$ ) was added to the surface-crosslinking solution and swelled for $10 \mathrm{~min}$. After removing the surface-crosslinking solution, the swollen CSAP was transferred to an aluminum tray and reacted at $170{ }^{\circ} \mathrm{C}$ for $15.0-25.0 \mathrm{~min}$. 
Table 2. Surface-crosslinking conditions.

\begin{tabular}{|c|c|c|c|c|c|}
\hline \multirow{3}{*}{ CSAP (g) } & \multicolumn{3}{|c|}{ Surface-Crosslinking Solution Composition } & \multirow{3}{*}{ Temperature $\left({ }^{\circ} \mathrm{C}\right)$} & \multirow{3}{*}{ Time (min) } \\
\hline & \multirow{2}{*}{ Methanol (g) } & \multirow{2}{*}{ DW (g) } & Surface-Crosslinker & & \\
\hline & & & BD (g) & & \\
\hline \multirow{7}{*}{3.0} & & & \multirow{7}{*}{1.0} & \multirow{7}{*}{170} & 15.0 \\
\hline & & & & & 17.5 \\
\hline & 6.1 & 2.9 & & & 20.0 \\
\hline & & & & & 22.5 \\
\hline & 5.9 & 3.1 & & & \multirow{3}{*}{17.5} \\
\hline & 6.3 & 2.7 & & & \\
\hline & 6.5 & 2.5 & & & \\
\hline
\end{tabular}

\subsection{Measurement}

\subsubsection{Fourier-Transform Infrared (FT-IR) Spectroscopic Analysis}

The structure of SAP was analyzed by Fourier-transform infrared spectroscopy with a resolution of $0.5 \mathrm{~cm}^{-1}$ (FT-IR, Nicolet iS10, Thermo Fisher Scientific, Waltham, MA, USA) using potassium bromide $(\mathrm{KBr})$ pellets in the scan range of $400-4000 \mathrm{~cm}^{-1}$.

\subsubsection{Free Absorbency (FA)}

SAP $(0.1 \mathrm{~g})$ was placed in a 100 mesh tea bag and swelled in excess distilled water at room temperature until the swelling equilibrium was reached. Then, the tea bag was taken out of the distilled water, excess water on the outside was wiped off, and it was weighed. In order to compare the absorption properties of SAP under various conditions, FA was measured in a $0.9 \mathrm{wt} \% \mathrm{NaCl}$ aqueous solution under the above conditions. A $0.9 \mathrm{wt} \%$ $\mathrm{NaCl}$ aqueous solution was used according to the international EDANA method (NWSP 240.0.R2), which has similar composition to human urine. FA is calculated as follows:

$$
F A=\frac{\omega_{1}-\omega_{0}}{\omega_{0}}
$$

where $\omega_{1}$ and $\omega_{0}$ are the weights of the swollen and dried SAP, respectively [37].

\subsubsection{Gel Content}

SAP $(0.5 \mathrm{~g})$ was immersed in $500 \mathrm{~mL}$ DW and stirred for $48 \mathrm{~h}$ to extract unreacted monomers and oligomers. After that, the SAP was screened and dried in an oven at $60{ }^{\circ} \mathrm{C}$ until there was no change in weight. The gel content is calculated as follows:

$$
\text { Gel content }=\frac{\omega_{e}}{\omega_{i}} \times 100
$$

where $\omega_{i}$ and $\omega_{e}$ are the weight of initial and extracted dried SAP, respectively [38].

\subsubsection{Centrifuge Retention Capacity (CRC)}

$\mathrm{CRC}$ is a value indicating how much water is retained after dewatering the swollen SAP with a centrifuge. SAP $(0.1 \mathrm{~g})$ was swollen in $0.9 \mathrm{wt} \% \mathrm{NaCl}$ aqueous solution for 30 min then dehydrated to $300 \mathrm{G}$ in a centrifuge and weighed according to EDANA method (NWSP 241.0.R2). CRC is calculated as follows:

$$
C R C=\frac{\omega_{2}-\omega_{1}}{\omega_{0}}
$$

where $\omega_{1}$ and $\omega_{0}$ are the weight of the swollen and dried SAP, respectively. 


\subsubsection{Absorbency Under Load (AUL)}

AUL is a value that indicates how much moisture the SAP can absorb under a given pressure. It is caused by the pressure applied to the SAP due to the infant's activity, and the average value of the load is taken as 0.3 psi according to EDANA method (NWSP 241.0.R2). As in the measurement of free absorbance, $200 \mathrm{~mL}$ of distilled water was poured into a beaker, and $\mathrm{NaCl}$ was added to prepare a $0.9 \mathrm{wt} \% \mathrm{NaCl}$ aqueous solution. A total of $0.16 \mathrm{~g}$ of the SAP was evenly distributed in the prepared cylinder, and the weight was taken using 0.3 psi weights. Then, a ceramic filter plate was placed on a chalet, and filter paper was placed on it. The cylinder containing SAP was placed on the filter paper, and sufficient aqueous solution of $\mathrm{NaCl}$ was added. After $1 \mathrm{~h}$, the cylinder was taken off, and the weight was measured with the weights. AUL is calculated as follows:

$$
A U L=\frac{\omega_{2}-\omega_{1}}{\omega_{0}}
$$

where $\omega_{1}$ and $\omega_{0}$ are the weight of the swollen and dried SAP, respectively (Figure 1).

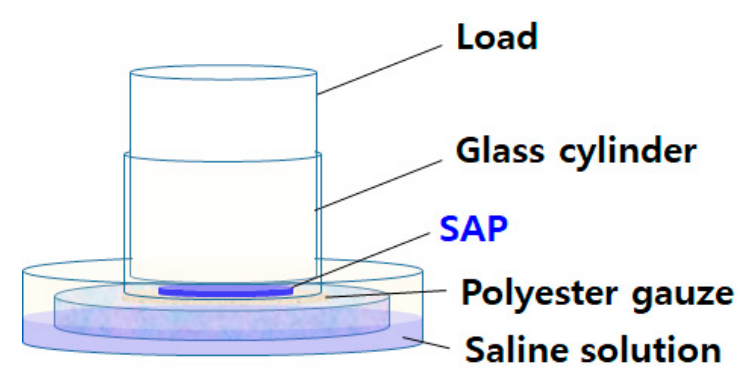

Figure 1. Scheme of the absorbency under load tester.

\subsubsection{Permeability}

Permeability is a measure of the amount of water flowing between swollen SAP layers. First, $0.5 \mathrm{~g}$ of SAP was added to the equipment and SAP was swollen for $30 \mathrm{~min}$ by adding a sufficient amount of $0.9 \mathrm{wt} \% \mathrm{NaCl}$ solution. A $0.3 \mathrm{psi}$ pressure device was installed on the swollen SAP. The cock at the bottom of the device was opened and the time taken for the $20 \mathrm{~mL} 0.9 \mathrm{wt} \% \mathrm{NaCl}$ solution to pass was measured.

\subsubsection{Rheological Properties}

The rheological measurements were performed using a rotational rheometer (TA instrument Ltd., ARES-G2, New Castle, DE, USA) with a parallel plate geometry (plate diameter of $25 \mathrm{~mm}$ and a gap of $3 \mathrm{~mm}$ ). The SAP sample $(0.2 \mathrm{~g})$ was added to $10 \mathrm{~mL}$ of DW and swelled for $30 \mathrm{~min}$. The rheological properties were measured at $25^{\circ} \mathrm{C}$ after placing the expanded gel particles on a parallel plate of a rheometer. The storage modulus $\left(G^{\prime}\right)$ and loss modulus $\left(G^{\prime \prime}\right)$ were recorded at constant shear strain $(0.2 \%)$ and a frequency ranging from $0.1-100 \mathrm{~Hz}$ [39].

\subsubsection{Degree of Biodegradation}

Permeability is a measure of the amount of water flowing between swollen SAP layers. First, $0.5 \mathrm{~g}$ of SAP was added to the equipment and SAP was swollen for $30 \mathrm{~min}$ by adding a sufficient amount of $0.9 \mathrm{wt} \% \mathrm{NaCl}$ solution. A 0.3 psi pressure device was installed on the swollen SAP. The cock at the bottom of the device was opened and the time taken for $20 \mathrm{~mL}$ of the $0.9 \mathrm{wt} \% \mathrm{NaCl}$ solution to pass was measured. For the degree of biodegradation test, the percentage biodegradation of ICVA SAPs were investigated according to ISO 14851:1999 and Chung-ang university laboratory method [40]. The microorganism solution was prepared by mixing solid solution diluted with standard media. Each sample, after freezing and drying, was mixed with microorganism solution in a BOD bottle. All sample bottles were kept in an incubator at $25^{\circ} \mathrm{C}$. 
The degree of biodegradation was calculated as follows:

$$
\text { Biodegradation }(\%)=\frac{B O D_{s}}{T h O D} \times 100
$$

where $T h O D$ is the theoretical oxygen demand in $\mathrm{mg} / \mathrm{g}$ of test material, $B O D_{S}$ is the specific BOD in $\mathrm{mg} / \mathrm{g}$ of test material, as shown in Equation (6):

$$
B O D_{s}=\frac{B O D_{t}-B O D_{b}}{P_{t}} .
$$

$B O D_{t}$ is the $\mathrm{BOD}$ of the flask containing the test material in $\mathrm{mg} / \mathrm{L}, B O D_{b}$ is the $\mathrm{BOD}$ of the blank flask in $\mathrm{mg} / \mathrm{L}$, and $P_{t}$ is the concentration of the test material in the flask in $\mathrm{mg} / \mathrm{L}$.

\section{Results and Discussion}

\subsection{Synthesis of ICVA CSAP}

For the CSAP synthesis, various monomers such as IA, cellulose, VSA, and AA were mixed in the water and added an internal crosslinking agents and initiator (Scheme 1). IA, VSA, and AA are highly hydrophilic materials due to hydroxyl groups, and radical polymerization is possible because of vinyl groups. The hydrogen atoms in the hydroxy groups in cellulose contribute to the formation of more active alkoxy radicals, while radical anions dissociated from the ammonium persulfate (APS) initiator react with vinyl monomers to form polymer chains, in which crosslinking occurred due to the internalized crosslinking agent. The monomer composition ratio of IA and AA was fixed at 50:40 and the ratio of cellulose and VSA was adjusted at the remaining $10 \%$. A total of $0.5 \mathrm{~g}$ of 1,6-hexanediol diacrylate (HDODA) and poly (ethylene glycol) diacrylate (PEGDA) at a 1:1 ratio as the surface-crosslinking agent was added along with $0.5 \mathrm{~g}$ of APS as the initiator (Table 1).
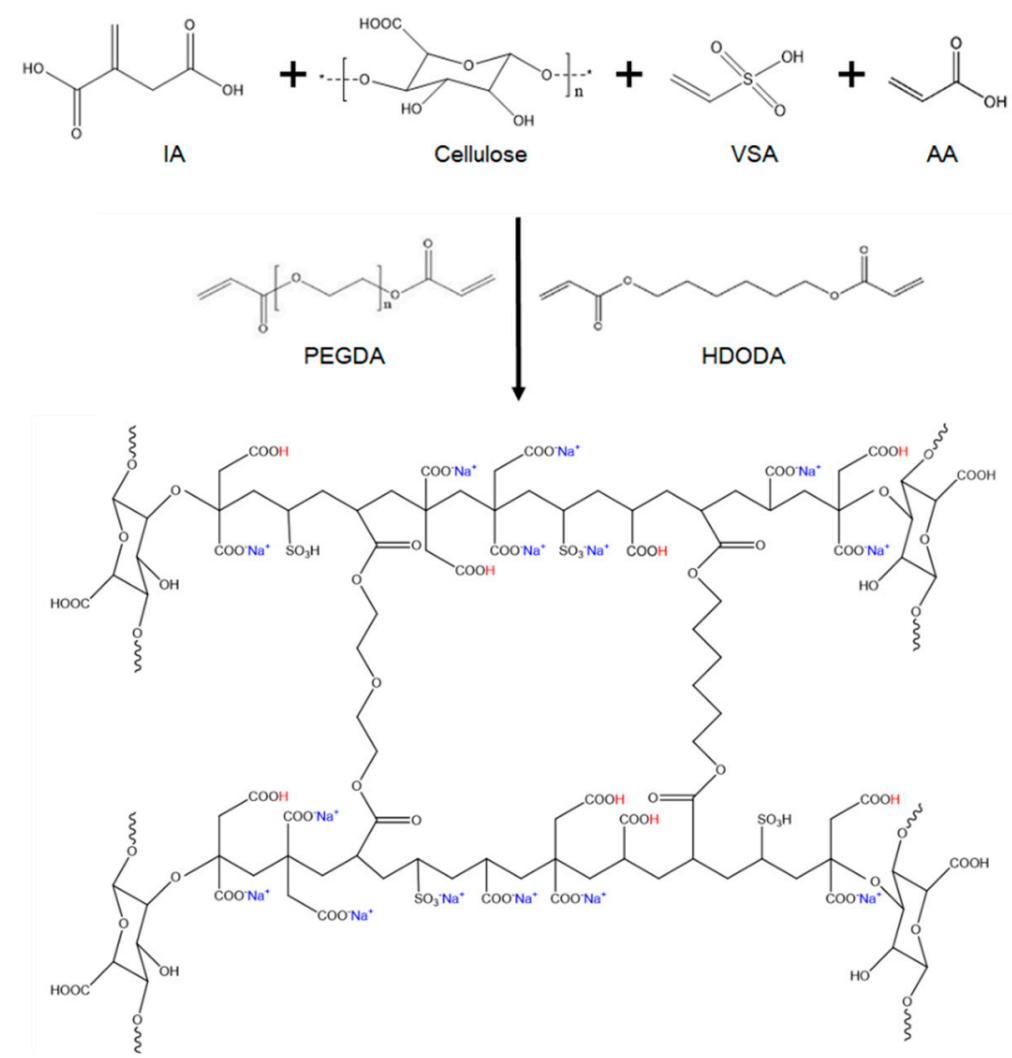

Scheme 1. Schematic synthesis of CSAP. 


\subsection{FT-IR Analysis of Monomers and IC 2.5 VA CSAP}

Figure 2 presents Fourier-transform infrared spectroscopy (FT-IR) spectra of IA, AA, VSA, cellulose, and $\mathrm{IC}_{2.5} \mathrm{VA}$ CSAP. The absorption band at $1721 \mathrm{~cm}^{-1}$ were attributed to the $\mathrm{C}=\mathrm{O}$ stretching vibrations of carboxyl groups $(-\mathrm{COOH})$ in IA and $\mathrm{AA}$, respectively. The peak at 819 and $1680 \mathrm{~cm}^{-1}$ indicates $\mathrm{C}=\mathrm{CH}_{2}$ of vinyl groups in IA, AA, and VSA. In $\mathrm{IC}_{2.5} \mathrm{VA}-\mathrm{CSAP}$, the -COO- peak was identified in the region around $1584 \mathrm{~cm}^{-1}$ due to the stable resonance effect of the neutralized carboxylic acid. In the spectrum of the cellulose, absorption peaks at $3550-3200 \mathrm{~cm}^{-1}$ correspond to $\mathrm{O}-\mathrm{H}$ stretching, while for VSA, peaks consistent with $\mathrm{S}=\mathrm{O}$ absorption at $1300 \mathrm{~cm}^{-1}$ and $-\mathrm{SO}_{2}-\mathrm{O}$ stretching vibration at $1184 \mathrm{~cm}^{-1}$ were evident. In $\mathrm{IC}_{2.5} \mathrm{VA}, \mathrm{C}-\mathrm{H}$ stretching (the same as in $\mathrm{AA}$ and IA) was seen at $2930 \mathrm{~cm}^{-1}$, and $\mathrm{C}-\mathrm{O}-\mathrm{C}$ and $\mathrm{C}-\mathrm{O}-\mathrm{H}$ stretching occurred at $1015 \mathrm{~cm}^{-1}$. The characteristic peaks of each monomer were observed in the $\mathrm{IC}_{2.5} \mathrm{VA}$ CSAP spectrum. In addition, the $\mathrm{C}=\mathrm{C}$ peaks appearing in IA, VSA, and AA disappeared by polymerization, which infers the successful synthesis of $\mathrm{IC}_{2.5} \mathrm{VA}$ CSAP.

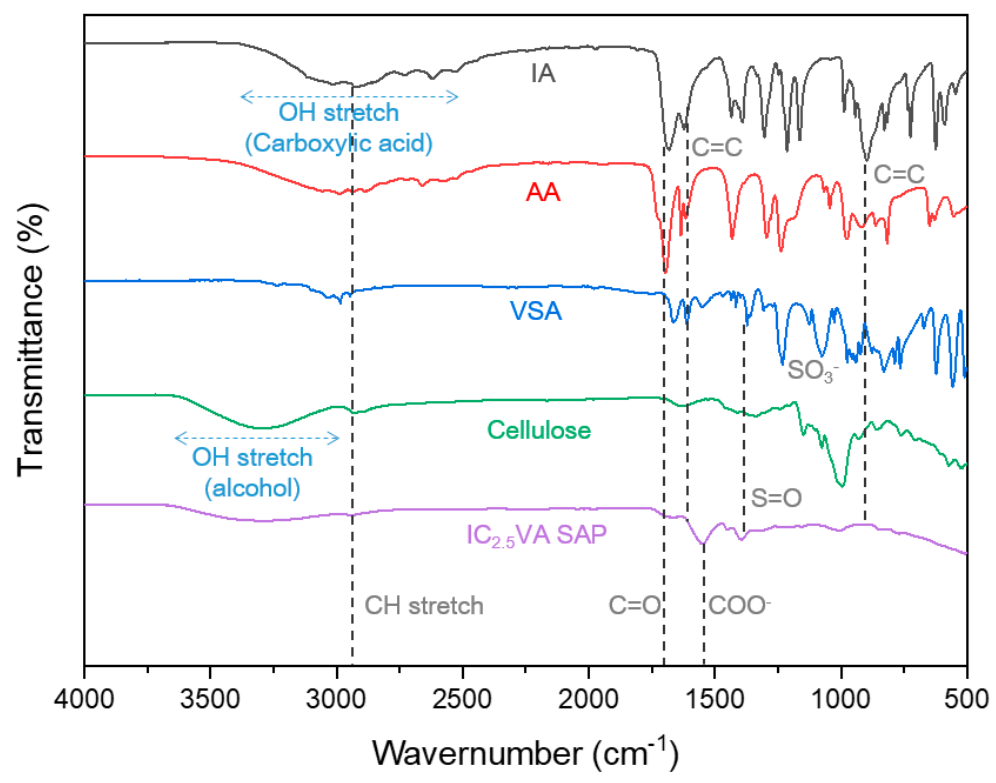

Figure 2. FT-IR spectra of IA, cellulose, VSA, AA, and $\mathrm{IC}_{2.5} \mathrm{VA}$ CSAP.

\subsection{Properties of SAPs According to Composition of Cellulose and VSA}

CRC and AUL values were compared according to CSAP composition (Table 3). Higher content of VSA has better CRC since VSA can absorb a large amount of water due to its high anionicity. Moreover, more cellulose improved the AUL due to its large surface area and porosity in the SAP structure.

Table 3. The centrifuge retention capacity (CRC) and absorbency under load (AUL) of the CSAP according to the composition of monomer.

\begin{tabular}{ccc}
\hline \multirow{2}{*}{ NAME } & \multicolumn{2}{c}{ CSAP } \\
\cline { 2 - 3 } & $\begin{array}{l}\text { CRC } \\
(\mathbf{g} / \mathbf{g})\end{array}$ & $\begin{array}{l}\text { AUL } \\
(\mathbf{g} / \mathbf{g})\end{array}$ \\
\hline $\mathrm{IVA}$ & 55.8 & 6.6 \\
\hline $\mathrm{IC}_{2.5} \mathrm{VA}$ & 52.3 & 7.6 \\
\hline $\mathrm{IC}_{5.0} \mathrm{VA}$ & 51.3 & 7.6 \\
\hline $\mathrm{IC}_{7.5} \mathrm{VA}$ & 49.8 & 7.3 \\
\hline $\mathrm{ICA}$ & 49.6 & 7.8 \\
\hline
\end{tabular}


In Figure 3, the IVA shows high free absorbency (FA) at all concentrations in $0.0-0.9 \mathrm{wt} \%$ $\mathrm{NaCl}$ solution and $454 \mathrm{~g} / \mathrm{g}$ in distilled water. $\mathrm{IC}_{2.5} \mathrm{VA}$ containing cellulose presented a high FA of $414 \mathrm{~g} / \mathrm{g}$ in distilled water and $92 \mathrm{~g} / \mathrm{g}$ in a $0.9 \mathrm{wt} \% \mathrm{NaCl}$ solution. Therefore, surface-crosslinking studies were conducted using $\mathrm{IC}_{2.5} \mathrm{VA}$, which has excellent $\mathrm{CRC}$, AUL, and FA values.

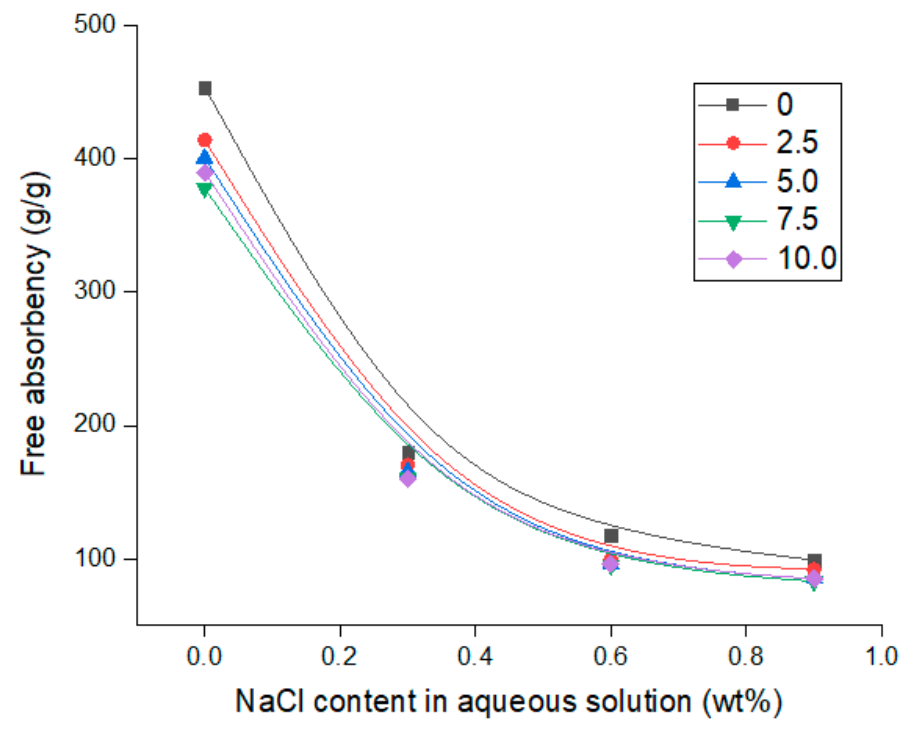

Figure 3. The free absorbency of the CSAP according to content of cellulose on various concentration of $\mathrm{NaCl}$ solution.

The synthesized ICVA CSAP was treated with surface-crosslinking agent 1,4-butanediol (BD) while mixing with water and methanol to improve the gel strength. Hydroxy functional groups in BD participated in esterification with the residual carboxyl groups in IA, cellulose, and AA. This reaction occurred irregularly on the ICVA CSAP surface and did not have much effect on the inside of the CSAP. However, it is important to select the appropriate amount of water in the surface-crosslinking solution because it is a significant factor in determining the depth at which BD penetrates into the CSAP. Deeper penetration increases the surface-crosslinking density and improves gel strength by reducing the FA value.

Table 4 compares the absorbency of the surface-crosslinked SAP (SSAP) according to the composition ratio of the comonomers. As the cellulose amount in the component ratio increased, CRC decreased while AUL increased. This showed a similar tendency after surface-crosslinking treatment, especially when $2.5 \mathrm{~g}$ cellulose was injected, which improved the AUL and permeability. Cellulose can have a positive effect on permeability because it has a large surface area relative to its molecular weight, has excellent dispersion in water, and can give high porosity to the SAP structure. Ali Olad et al. reported that cellulose can provide pores in SAP because it forms physical crosslinking points between polymer chain and grafted polymeric structure. In addition, physical crosslinking density increases in the presence of cellulose due to the generation of additional hydrogen-bonding interactions within the matrix, which results in the formation of stiffer gel network structure with higher gel strength [41]. During surface-crosslinking treatment, the hydroxyl groups of cellulose can form a more robust surface through esterification, which can have a positive effect on AUL, permeability, and gel content. Moreover, the SSAP had $90 \%$ good gel content, although no tendency was prevalent in the composition. 
Table 4. The absorption properties of the surface-crosslinked SAP (SSAP) according to the composition of monomer.

\begin{tabular}{ccccc}
\hline \multirow{2}{*}{ CSAP } & \multicolumn{3}{c}{ SSAP * } \\
\cline { 2 - 5 } & $\begin{array}{c}\text { CRC } \\
(\mathbf{g} / \mathbf{g})\end{array}$ & $\begin{array}{c}\text { AUL } \\
(\mathbf{g} / \mathbf{g})\end{array}$ & $\begin{array}{c}\text { Permeability } \\
(\mathbf{s e c})\end{array}$ & $\begin{array}{c}\text { Gel Content } \\
(\mathbf{\%})\end{array}$ \\
\hline IVA & 30.6 & 17.4 & 126 & 90.0 \\
\hline $\mathrm{IC}_{2.5} \mathrm{VA}$ & 29.0 & 22.8 & 42 & 90.6 \\
\hline $\mathrm{IC}_{5.0} \mathrm{VA}$ & 27.0 & 22.9 & 35 & 90.1 \\
\hline $\mathrm{IC}_{7.5} \mathrm{VA}$ & 25.5 & 24.1 & 25 & 89.2 \\
\hline ICA & 24.4 & 23.6 & 28 & 89.4
\end{tabular}

* SSAP synthesis conditions: (a) CSAP $=3 \mathrm{~g}$, (b) temperature $=170^{\circ} \mathrm{C}$, (c) reaction time $=20 \mathrm{~min}$, and (d) weight ratio of methanol, $\mathrm{DW}$, and $\mathrm{BD}=6.1: 2.9: 1.0$.

We measured the storage modulus and the loss modulus in order to compare the gel strength of CSAP and SSAP using $\mathrm{IC}_{2.5} \mathrm{VA}$ samples. Flory-Rehner et al. suggested that the crosslinking density of a polymer is directly related to its storage modulus [38]. As shown in Figure 4, we confirmed that the storage modulus of $\mathrm{IC}_{2.5}$ VA SSAP was significantly higher than $\mathrm{IC}_{2.5}$ VA CSAP. Accordingly, we were able to predict that the gel strength of CSAP was improved by surface-crosslinking.

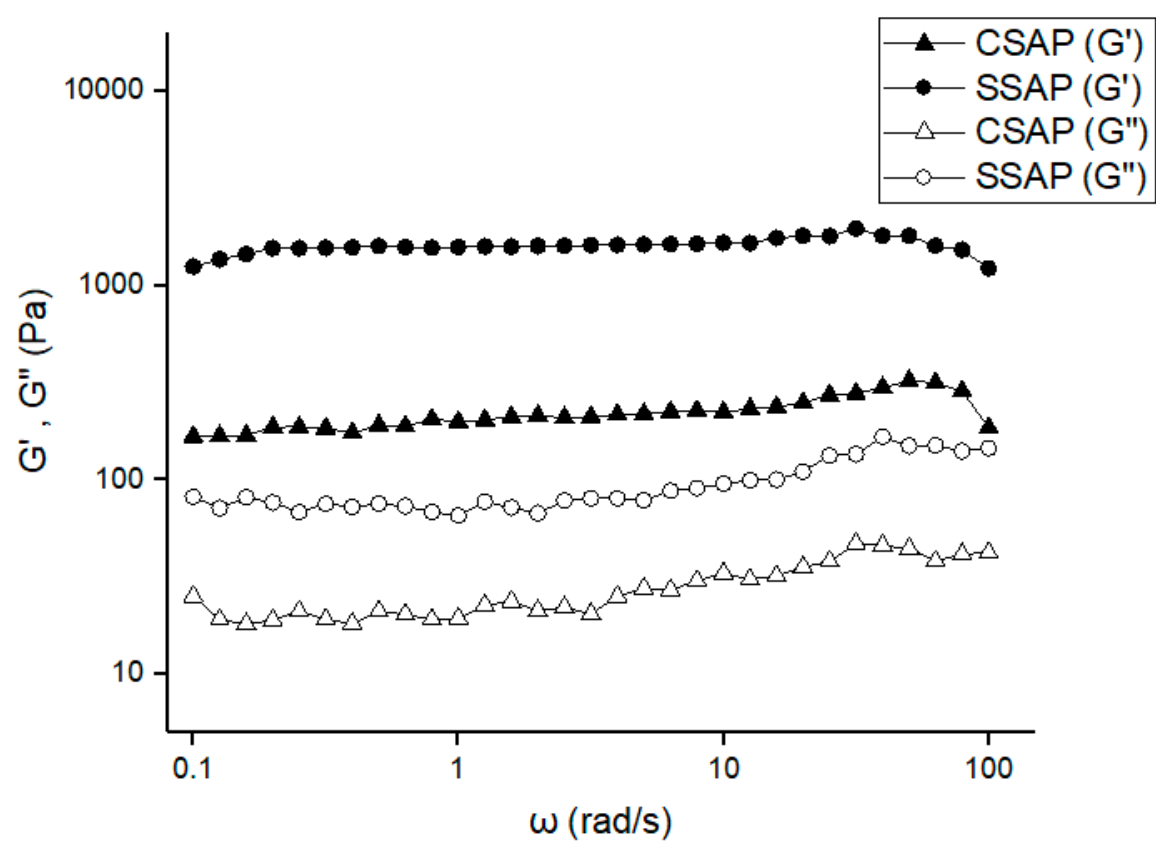

Figure 4. $\mathrm{G}^{\prime}$ and $\mathrm{G}^{\prime \prime}$ frequency of $\mathrm{IC}_{2.5} \mathrm{VA} \mathrm{CSAP}$ and SSAP.

Figure 5 exhibits the results of a comparative study into the biodegradability of $\mathrm{IC}_{2.5} \mathrm{VA}$ and a hydrogel manufactured from $100 \%$ biodegradable cellulose as the comparator. The results confirm that CSAP was more easily biodegraded than SSAP. When compared to the relative biodegradability of cellulose hydrogel (based on 100\%) after 182 days (26 weeks), CSAP and SSAP had biodegraded by $85 \%$ and $71 \%$, respectively. Hence, the increase in crosslinking density by the surface-crosslinking treatment reduced biodegradability, although it was still acceptable. Using $\mathrm{IC}_{2.5} \mathrm{VA}$, which showed excellent biodegradability and absorbency in the previous experiments, the performance changes were additionally compared with the surface-crosslinking time and solution composition. 


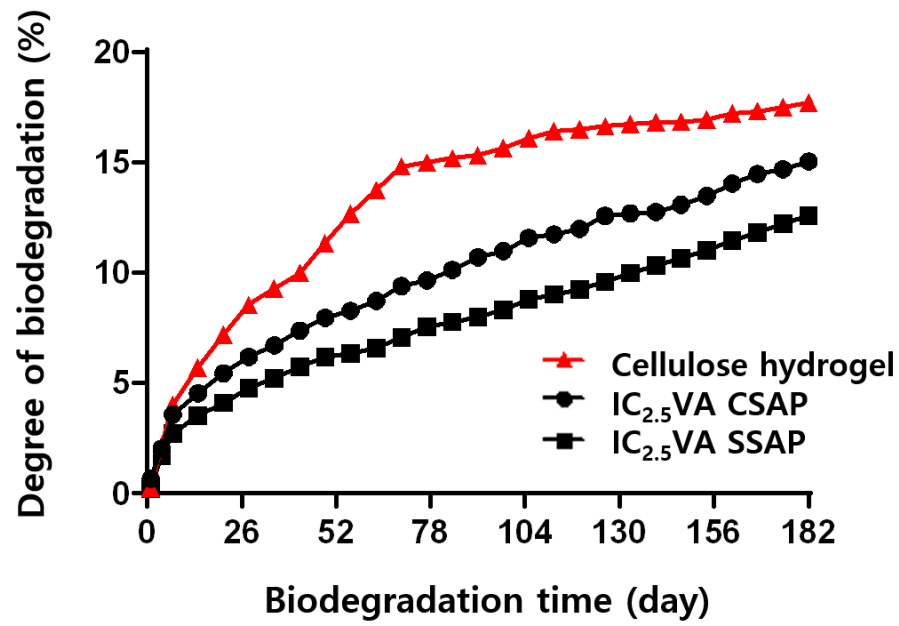

Figure 5. Degree of biodegradation of $\mathrm{IC}_{2.5} \mathrm{VA}$ CSAP and SSAP.

\subsection{Properties of SAPs According to Surface-Crosslinking Time and Composition of} Surface-Crosslinking Solution

In the conditions reported in Table 2, the performance of CRC, AUL, and permeability over surface-crosslinking time are compared in Figure 6. The ICVA CSAP synthesized in this study was very sensitive to temperature changes because of it having the highest amount of IA, which has low thermal resistance in the composition ratio. Temperatures above $170{ }^{\circ} \mathrm{C}$ or more than 25 min of surface-crosslinking time caused degradation in gel performance due to the thermal decomposition of CSAP. As the surface-crosslinking time increased, CRC decreased whereas AUL and permeability performance increased. In particular, excellent CRC values and decent AUL and permeability values were obtained at 17.5 min of surface-crosslinking time. According to Lee et al., the surface-crosslinked layer thickens as the surface-crosslinking time increases [34]. Nevertheless, the AUL and permeability values could be improved by changing the surface-crosslinking thickness by adjusting the amount of water in the surface-crosslinking solution.

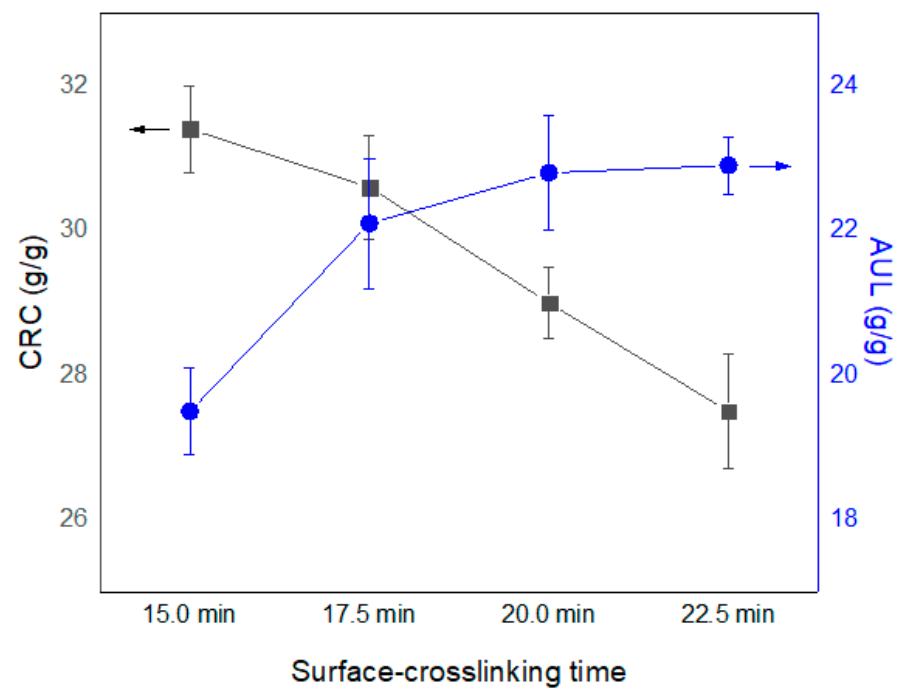

(a)

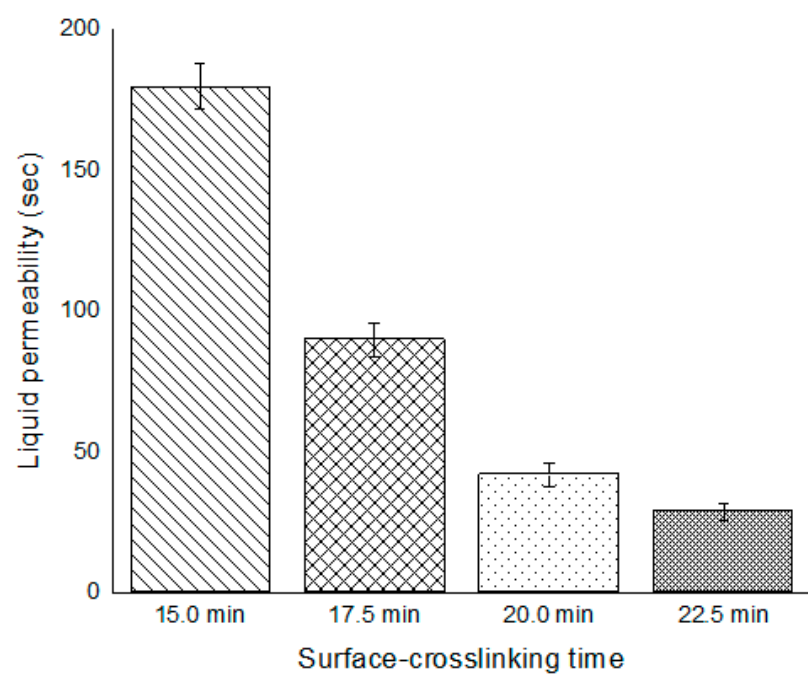

(b)

Figure 6. CRC and AUL (a) and permeability (b) of the SSAP according to surface-crosslinking time.

Distilled water in the surface-crosslinking solution penetrates the surface of the CSAP. Increased thickness of the surface-crosslinked layer can reduce absorption performance in terms of CRC and FA because it interferes with the expansion of CSAP. When the distilled 
water content of less than $2.7 \mathrm{~g}$ was injected, the CRC value was above $36 \mathrm{~g} / \mathrm{g}$ but the AUL and permeability values were drastically reduced (Figure 7). On the other hand, when the distilled water content was $3.1 \mathrm{~g}$, CRC was more than $30 \mathrm{~g} / \mathrm{g}$, AUL was $23 \mathrm{~g} / \mathrm{g}$, and the permeability was $55 \mathrm{~s}$, which are results that indicate excellent performance.

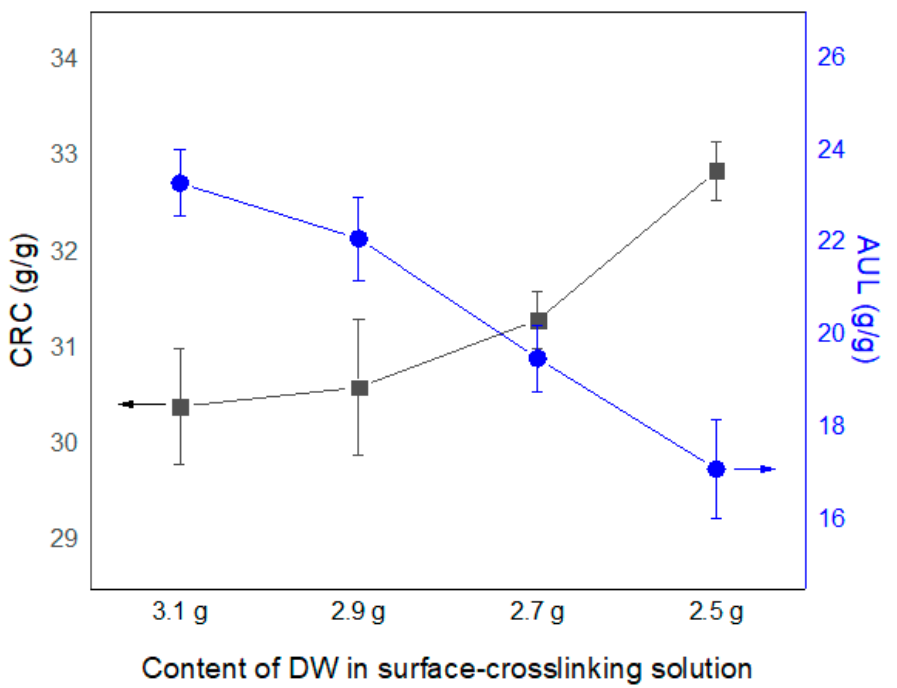

(a)

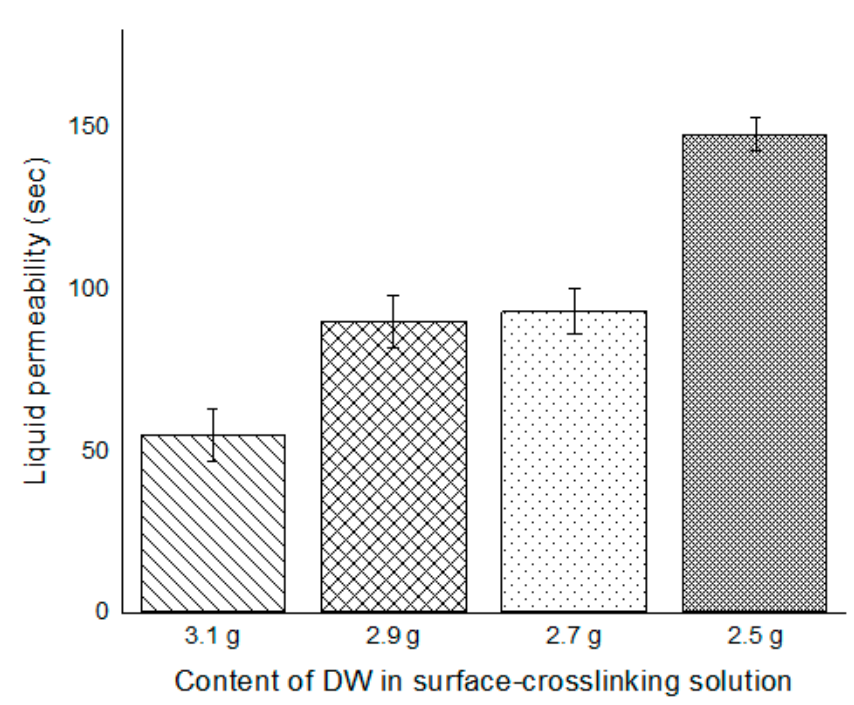

(b)

Figure 7. The CRC and AUL (a) and permeability (b) of the SSAP according to content of DW in surface-crosslinking solution.

In Figure 8, the biodegradability of CSAP is compared with the cellulose hydrogel (the reference specimen) over 112 days (16 weeks) with the distilled water content in the 2.7-3.1 g range. It was found that the crosslinked surface layer was thinner and improved biodegradability as the distilled water content decreased. Compared to the biodegradability of the cellulose hydrogel, $\mathrm{IC}_{2.5} \mathrm{VA}$ (DW_2.7 g) showed a relative biodegradability of $63 \%$, and $\mathrm{IC}_{2.5} \mathrm{VA}$ (DW_3.1 g) showed a relative biodegradability of $54 \%$. The optimum surfacecrosslinking treatment conditions for the surface-crosslinking time and the amount of distilled water in the surface-crosslinking solution increased the gel strength by solidifying the surface of the CSAP but reduced the biodegradability. However, this was observing the biodegradation over a relatively short period of time, and when biodegradation was allowed to continue, the improved gel strength of the ICVA coincided with acceptable biodegradability.

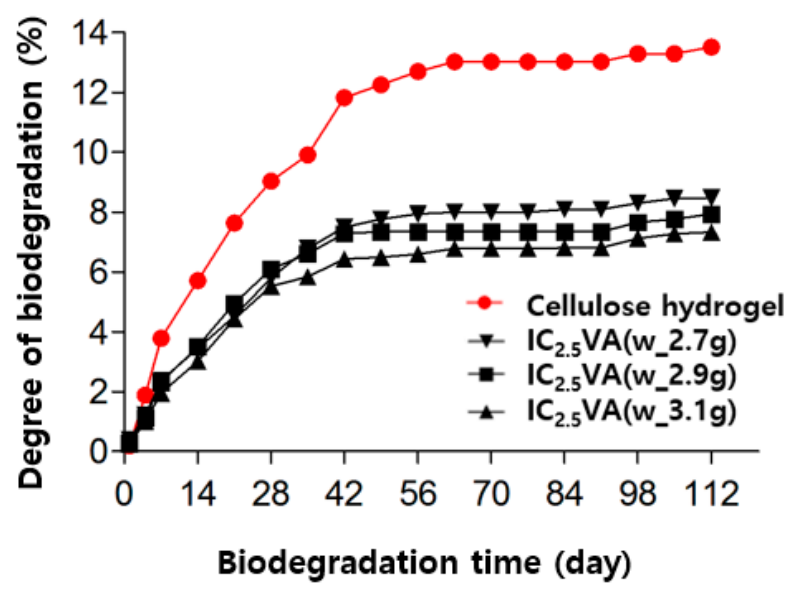

Figure 8. Degree of biodegradation according to water content in surface-crosslinking solution. 


\section{Conclusions}

Our research is based on the newly designed eco-friendly biodegradable SAP made of biomass-derived IA and cellulose. The absorption performance of other eco-friendly SAPs is significantly inferior to the existing commercial AA based SAP. On the other hand, we prepared a SAP with almost similar performance of commercial SAP used in hygienic diapers. ICVA CSAP was synthesized using IA, cellulose, VSA, and AA, the successful synthesis of which was confirmed through FT-IR analysis. After surface-crosslinking treatment, the gel content was maintained at around $90 \%$. As a result of adjusting the composition of cellulose and VSA, the FA and CRC of CSAP decreased but the AUL slightly increased as the cellulose component was increased. These results were more pronounced in the SSAP and were particularly effective in improving gel strength. Hence, $\mathrm{IC}_{2.5} \mathrm{VA}$ with appropriate CRC, AUL, and permeability was selected as the optimal composition ratio, and the biodegradability of CSAP and SSAP was compared.

The study of surface-crosslinking treatment time adjustment using $\mathrm{IC}_{2.5} \mathrm{VA}$ confirmed that AUL and permeability improved significantly as the surface-crosslinking treatment time increased. However, the CRC decreased sharply after $20 \mathrm{~min}$, leading to a shorter time (17.5 $\mathrm{min}$ ) being selected. Furthermore, the performance was supplemented by adjusting the amount of distilled water in the surface-crosslinking solution. When the amount of distilled water was increased from 2.9 to $3.1 \mathrm{~g}$, the AUL and permeability were improved.

It was expected that the increase of the distilled water content in the surface-crosslinking solution facilitated penetration of the surface-crosslinking agent, thereby increasing the thickness of the surface-crosslinking layer.

The successfully developed SSAP with $54 \%$ relative biodegradability compared to standard cellulose hydrogel (100\% biodegradable), CRC $30.4 \mathrm{~g} / \mathrm{g}$, AUL $23.3 \mathrm{~g} / \mathrm{g}$, and permeability of $55 \mathrm{~s}$ could be a commercial replacement for existing SAPs comprising petroleumderived raw materials.

Author Contributions: Conceptualization, D.H.K. and Y.S.L.; methodology, J.S.K. and Y.S.L.; investigation, J.S.K. and D.H.K.; writing—original draft preparation, J.S.K.; writing—review and editing, J.S.K. and Y.S.L.; visualization, J.S.K. All authors have read and agreed to the published version of the manuscript.

Funding: This research received no external funding.

Data Availability Statement: Data sharing not applicable.

Acknowledgments: This work was supported by the Technology Innovation Program (Parts and Materials Technology Development) (20010106, Adhesives with low water permeability and low outgassing) funded By the Ministry of Trade, Industry \& Energy (MOTIE, Republic of Korea).

Conflicts of Interest: The authors declare that there are no conflicts of interest regarding the publication of this paper.

\section{References}

1. Ahmed, E.M. Hydrogel: Preparation, characterization and applications: A review. J. Adv. Res. 2015, 6, 105-121. [CrossRef]

2. Zhou, L.; Cai, D.; He, L.; Zhong, N.; Yu, M.; Zhang, X.; Wu, Z. Fabrication of a high-performance fertilizer to control the loss of water and nutrient using micro/nano networks. ACS Sustain. Chem. Eng. 2015, 3, 645-653. [CrossRef]

3. Schmaljohann, D. Thermo-and pH-responsive polymers in drug delivery. Adv. Drug Deliv. Rev. 2006, 58, 1655-1670. [CrossRef] [PubMed]

4. Lee, S.G.; Kim, M.Y.; Lee, J.M.; Jang, M.S. Superabsorbent Polymer. U.S. Patent No. 9,950,308, 24 April 2018.

5. Hwang, K.S.; Jang, S.S.; Jung, Y.W.; Lee, S.H.; Ha, K.R. Studies on the Strength of Cement Mortars with Surface Crosslinked cPSA Absorbent. Polym. Korea 2012, 36, 208-215. [CrossRef]

6. Azad, M.M.; Sandros, M.G. Microwave-assisted polymerization: Inert addition and surface coating of superabsorbent polymer for improved physical properties. J. Appl. Polym. Sci. 2016, 133, 43990. [CrossRef]

7. Chang, S.; Kim, M.; Oh, S.; Min, J.H.; Kang, D.; Han, C.; Lee, H. Multi-scale characterization of surface-crosslinked superabsorbent polymer hydrogel spheres. Polymer 2015, 145, 174-183. [CrossRef]

8. Mojarad-Jabali, S.; Kabiri, K.; Karami, Z.; Mastropietro, D.J.; Omidian, H. Surface cross-linked SAPs with improved swollen gel strength using diol compounds. J. Macromol. Sci. A 2020, 57, 62-71. [CrossRef] 
9. Xu, X.; Bai, B.; Ding, C.; Wang, H.; Suo, Y. Synthesis and properties of an ecofriendly superabsorbent composite by grafting the poly(acrylic acid) onto the surface of dopamine-coated sea buckthorn branches. Ind. Eng. Chem. Res. 2015, 54, 3268-3278. [CrossRef]

10. Huang, Y.; Zeng, M.; Ren, J.; Wang, J.; Fan, L.; Xu, Q. Preparation and swelling properties of graphene oxide/poly (acrylic acid-co-acrylamide) super-absorbent hydrogel nanocomposites. Colloids Surf. A Physicochem. Eng. Asp. 2012, 401, 97-106. [CrossRef]

11. Maitra, J.; Shukla, V.K. Cross-linking in hydrogels-a review. Am. J. Polym. Sci. 2014, 4, 25-31.

12. Karadağ, E.; Saraydın, D.; Güven, O. Radiation induced superabsorbent hydrogels. Acrylamide/itaconic acid copolymers. Macromol. Mater. Eng. 2001, 286, 34-42.

13. Dabbaghi, A.; Jahandideh, A.; Kabiri, K.; Ramazani, A.; Zohuriaan-Mehr, M.J. The synthesis and incorporation of a star-shaped biobased modifier in the acrylic acid based superabsorbent: A strategy to enhance the absorbency under load. Polym.-Plast. Tech. Mat. 2019, 58, 1678-1690. [CrossRef]

14. Bhuyan, M.; Chandra Dafader, N.; Hara, K.; Okabe, H.; Hidaka, Y.; Rahman, M.; Rahman, N. Synthesis of potato starch-acrylic-acid hydrogels by gamma radiation and their application in dye adsorption. Int. J. Polym. Sci. 2016, 2016, 1-11. [CrossRef]

15. Ma, M.; Mukerabigwi, J.F.; Huang, R.; Lei, S.; Huang, X.; Cao, Y. Eco-Friendly Superabsorbent Synthesis Based on Polysaccharides. J. Polym. Environ. 2020, 28, 2801-2809. [CrossRef]

16. Czarnecka, E.; Nowaczyk, J. Semi-Natural Superabsorbents Based on Starch-g-poly (acrylic acid): Modification, Synthesis and Application. Polymers 2020, 12, 1794. [CrossRef] [PubMed]

17. Willke, T.; Vorlop, K.D. Biotechnological production of itaconic acid. Appl. Microbiol. Biotechnol. 2001, 56, 289-295. [CrossRef]

18. Lanthong, P.; Nuisin, R.; Kiatkamjornwong, S. Graft copolymerization, characterization, and degradation of cassava starch-gacrylamide/itaconic acid superabsorbents. Carbohydr. Polym. 2006, 66, 229-245. [CrossRef]

19. Yang, J.; Li, F.; Li, M.; Zhang, S.; Liu, J.; Liang, C.; Xiong, L. Fabrication and characterization of hollow starch nanoparticles by gelation process for drug delivery application. Carbohydr. Polym. 2017, 173, 223-232. [CrossRef]

20. Mylangam, C.K.; Beeravelli, S.; Medikonda, J.; Pidaparthi, J.S.; Kolapalli, V.R.M. Badam gum: A natural polymer in mucoadhesive drug delivery. Design, optimization, and biopharmaceutical evaluation of badam gum-based metoprolol succinate buccoadhesive tablets. Drug Deliv. 2016, 23, 195-206. [CrossRef]

21. Riyajan, S.A. Robust and biodegradable polymer of cassava starch and modified natural rubber. Carbohydr. Polym. 2015, 134, 267-277. [CrossRef]

22. Zhong, K.; Lin, Z.T.; Zheng, X.L.; Jiang, G.B.; Fang, Y.S.; Mao, X.Y.; Liao, Z.W. Starch derivative-based superabsorbent with integration of water-retaining and controlled-release fertilizers. Carbohydr. Polym. 2013, 92, 1367-1376. [CrossRef] [PubMed]

23. Qiao, D.; Liu, H.; Yu, L.; Bao, X.; Simon, G.P.; Petinakis, E.; Chen, L. Preparation and characterization of slow-release fertilizer encapsulated by starch-based superabsorbent polymer. Carbohydr. Polym. 2016, 147, 146-154. [CrossRef] [PubMed]

24. Qiao, D.; Tu, W.; Wang, Z.; Yu, L.; Zhang, B.; Bao, X.; Lin, Q. Influence of crosslinker amount on the microstructure and properties of starch-based superabsorbent polymers by one-step preparation at high starch concentration. Int. J. Biol. Macromol. 2019, 129, 679-685. [CrossRef] [PubMed]

25. Kim, S.J.; Park, S.J.; Kim, S.I. Properties of smart hydrogels composed of polyacrylic acid/poly (vinyl sulfonic acid) responsive to external stimuli. Smart Mater. Struct. 2004, 13, 317. [CrossRef]

26. Anirudhan, T.S.; Rejeena, S.R. Poly (methacrylic acid-co-vinyl sulfonic acid)-grafted-magnetite/nanocellulose superabsorbent composite for the selective recovery and separation of immunoglobulin from aqueous solutions. Sep. Purif. Technol. 2013, 119, 82-93. [CrossRef]

27. Pourjavadi, A.; Ghasemzadeh, H. Carrageenan-g-poly (acrylamide)/poly (vinylsulfonic acid, sodium salt) as a novel semi-IPN hydrogel: Synthesis, characterization, and swelling behavior. Polym. Eng. Sci. 2007, 47, 1388-1395. [CrossRef]

28. Hussain, T.; Ansari, M.; Ranjha, N.M.; Khan, I.U.; Shahzad, Y. Chemically cross-linked poly (acrylic-co-vinylsulfonic) acid hydrogel for the delivery of isosorbide mononitrate. Sci. World J. 2013, 2013, 1-9. [CrossRef]

29. Karami, Z.; Naderi, P.; Kabiri, K.; Zohuriaan-Mehr, M.J. Epoxidized and Cyclocarbonated Star-Shaped Macromolecules as Bio-Based Internal and External Crosslinkers for Superabsorbent Polymer Hydrogels. J. Polym. Environ. 2020, 28, 1684-1694. [CrossRef]

30. Moini, N.; Kabiri, K.; Zohuriaan-Mehr, M.J. Practical improvement of SAP hydrogel properties via facile tunable cross-linking of the particles surface. Polym.-Plast. Tech. Eng. 2016, 55, 278-290. [CrossRef]

31. Azizi, A.; Kabiri, K.; Zohuriaan-Mehr, M.J.; Bouhendi, H.; Karami, Z. Transamidation: A feasible approach of surface modification to improve absorbency under load of agricultural superabsorbent materials. J. Mater. Res. 2018, 33, 2327-2335. [CrossRef]

32. Ghasri, M.; Bouhendi, H.; Kabiri, K.; Zohuriaan-Mehr, M.J.; Karami, Z.; Omidian, H. Superabsorbent polymers achieved by surface cross linking of poly (sodium acrylate) using microwave method. Iran. Polym. J. 2019, 28, 539-548. [CrossRef]

33. Ha, J.; Kim, M.; Lee, W.; Lee, H.; Han, C.; Koh, W.G.; Ryu, D.Y. Direct measurement of crosslinked surface layer in superabsorbent poly (acrylic acid). Mater. Lett. 2018, 228, 33-36. [CrossRef]

34. Lee, K.M.; Min, J.H.; Oh, S.; Lee, H.; Koh, W.G. Preparation and characterization of superabsorbent polymers (SAPs) surfacecrosslinked with polycations. React. Funct. Polym. 2020, 157, 104774. [CrossRef]

35. Yadav, M.; Srivastav, A.; Verma, S.K.; Behari, K. Graft (partially carboxymethylated guar gum-g-poly vinyl sulfonic acid) copolymer: From synthesis to applications. Carbohydr. Polym. 2013, 97, 597-603. [CrossRef] 
36. Chen, M.; Ni, Z.; Shen, Y.; Xiang, G.; Xu, L. Reinforced swelling and water-retention properties of super-absorbent hydrogel fabricated by a dual stretchable single network tactic. Colloids Surf. A Physicochem. Eng. Asp. 2020, 608, 125133. [CrossRef]

37. Pourjavadi, A.; Amini-Fazl, M.S.; Ayyari, M. Optimization of synthetic conditions CMC-g-poly (acrylic acid)/Celite composite superabsorbent by Taguchi method and determination of its absorbency under load. Express Polym. Lett. 2007, 1, 488-494. [CrossRef]

38. Pulat, M.; Eksi, H. Determination of swelling behavior and morphological properties of poly (acrylamide-co-itaconic acid) and poly (acrylic acid-co-itaconic acid) copolymeric hydrogels. J. Appl. Polym. Sci. 2006, 102, 5994-5999. [CrossRef]

39. Dabbaghi, A.; Kabiri, K.; Ramazani, A.; Zohuriaan-Mehr, M.J.; Jahandideh, A. Synthesis of bio-based internal and external cross-linkers based on tannic acid for preparation of antibacterial superabsorbents. Polym. Adv. Technol. 2019, 30, $2894-2905$. [CrossRef]

40. Amonpattaratkit, P.; Khunmanee, S.; Kim, D.H.; Park, H. Synthesis and characterization of gelatin-based crosslinkers for the fabrication of superabsorbent hydrogels. Materials 2017, 10, 826. [CrossRef]

41. Olad, A.; Doustdar, F.; Gharekhani, H. Fabrication and characterization of a starch-based superabsorbent hydrogel composite reinforced with cellulose nanocrystals from potato peel waste. Colloid Surf. A-Physicochem. Eng. Asp. 2020, 601, 124962. [CrossRef] 